\title{
BMJ Open Efficacy and safety of lapatinib and trastuzumab for HER2-positive breast cancer: a systematic review and meta- analysis of randomised controlled trials
}

\author{
Zhi-qiao Xu, Yan Zhang, Ning Li, Pei-jie Liu, Ling Gao, Xin Gao, Xiao-jing Tie
}

To cite: Xu Z-qiao, Zhang Y, Li N, et al. Efficacy and safety of lapatinib and trastuzumab for HER2-positive breast cancer: a systematic review and meta-analysis of randomised controlled trials. BMJ Open 2017;7:e013053. doi:10.1136/bmjopen-2016013053

- Prepublication history and additional material is available. To view please visit the journal (http://dx.doi.org/ 10.1136/bmjopen-2016013053).

Received 16 June 2016 Revised 18 January 2017 Accepted 19 January 2017

CrossMark

Tumor Center, Kaifeng Central Hospital, Kaifeng, China

Correspondence to Dr Yan Zhang;

zhang_yan_2016@163.com

\section{ABSTRACT}

Objectives: The anti-HER2 monoclonal antibody trastuzumab and the tyrosine kinase inhibitor lapatinib have complementary mechanisms of action and synergistic antitumour actively in models of HER2positive breast cancer. However, the efficacy of trastuzumab in combination with lapatinib remains controversial. Therefore, we conducted this metaanalysis to compare combination treatment with lapatinib and trastuzumab to trastuzumab or lapatinib alone in the treatment of HER2-positive breast cancer. Methods: Randomised controlled trials (RCTs), published in PubMed, Embase and Web of Science, were systematically reviewed to assess the survival benefits and toxicity profile of HER2-positive patients with breast cancer who were treated with lapatinib and trastuzumab. Outcomes included pathological complete response (pCR), event-free survival (EFS), overall survival (OS) and toxicities. Results were expressed as the risk ratio (RR) or HR with $95 \%$ Cls. Pooled estimates were calculated by using a fixed-effects model or a randomised-effects model. Results: A total of 7 RCTs involving 2084 patients met the inclusion criteria and were included in this metaanalysis. The combination of lapatinib and trastuzumab significantly improved $p C R(R R=1.43,95 \% \mathrm{Cl} 1.23$ to $1.67 ; p<0.001$ ), EFS ( $H R=0.75,95 \% \mathrm{Cl} 0.60$ to 0.93 ; $\mathrm{p}=0.009$ ) and $\mathrm{OS}$ ( $\mathrm{HR}=0.72,95 \% \mathrm{Cl} 0.56$ to 0.93 ; $\mathrm{p}=0.011$ ) in the treatment of HER2-positive breast cancer compared with trastuzumab or lapatinib alone. The combination treatment also increased the $\mathrm{pCR}$ irrespective of hormone receptor status and tumour size. More frequent grade 3 or 4 adverse events, including diarrhoea, rash or erythema, neutropenia and hepatic adverse events, were found in the combination group than in the trastuzumab or lapatinib group.

Conclusions: On the basis of the current evidence, our results reveal that the addition of lapatinib to

trastuzumab can significantly improve pCR, EFS and OS with a tolerated toxicity in patients with HER2-positive breast cancer. Further well-conducted, large-scale trials are needed to validate these findings.

\section{INTRODUCTION}

Amplification of the human epidermal growth factor receptor 2 (HER2) gene occurs

\section{Strengths and limitations of this study}

- This is a systematic review and meta-analysis of randomised controlled trials (RCTs) to compare combination treatment with lapatinib and trastuzumab to trastuzumab or lapatinib alone in the treatment of HER2-positive breast cancer.

- A total of 7 RCTs involving 2084 patients met the inclusion criteria and were included in this meta-analysis. All these studies included were high-quality, well-performed trials.

- We found that a combination of lapatinib and trastuzumab significantly improved pathological complete response ( $p C R$ ), event-free survival and overall survival in the treatment of HER2-positive breast cancer compared with trastuzumab or lapatinib alone. The combination treatment also increased the $\mathrm{PCR}$ irrespective of hormone receptor status and tumour size.

- Owing to the limited number of included studies and small sample size, the treatment effects of combination treatment with lapatinib and trastuzumab might be overestimated. Therefore, physicians should interpret our findings with caution when applying them in clinical practice.

in $\sim 20-25 \%$ of primary breast cancers, and is associated with poor clinical outcomes in the absence of systemic therapy. ${ }^{1}{ }^{2}$ An anti-HER2 drug such as the monoclonal antibody trastuzumab (herceptin, Genentech, San Francisco, California, USA) is widely used in the treatment of HER2-positive breast cancer. ${ }^{3}$ When used in combination with chemotherapy, trastuzumab significantly prolongs overall survival (OS) in patients with either metastatic disease ${ }^{3}$ or early breast cancer $^{4}{ }^{5}$ compared with chemotherapy alone. When used as an add-on to chemotherapy in the neoadjuvant setting, trastuzumab improves the pathological complete response $(\mathrm{pCR})$ rate $^{67}$ and event-free survival (EFS). ${ }^{7}$ Another anti-HER2 agent, the tyrosine kinase inhibitor lapatinib (tykerb, GlaxoSmithKline, Brentford, UK), has been 
approved for the treatment of patients with advanced HER2-positive breast cancer. ${ }^{8}$

Lapatinib prolonged progression-free survival (PFS) in patients who have progressed on trastuzumab when it was administered as monotherapy ${ }^{9} 10$ or in combination with trastuzumab, ${ }^{11}$ or with capecitabine. ${ }^{12}$

Since trastuzumab with lapatinib have complementary mechanisms in the treatment of tumours, it is possible that their combined use would produce better effects than either agent alone. Preclinical studies indicated that the combination treatment could improve apoptosis $^{13}$ and enhance tumour control in HER2-positive xenografts. ${ }^{14}$ In the NeoALTTO trial, ${ }^{15}$ the authors evaluated the effects of combination treatment with lapatinib and trastuzumab in HER2-positive breast cancers. Their results showed that the pCR rate in the combination group $(51.3 \%)$ was almost twofold higher than that in the trastuzumab group $(29.5 \%),{ }^{15}$ whereas in another large-scale randomised controlled trial (RCT) of NSABP protocol B- $41,{ }^{16}$ they also observed a trend towards improvement in pCR, but the trend was not statistically significant. ${ }^{16}$ Given that multiple trials observed controversial clinical outcomes of the combination therapy, it is vital to understand the full impact of the dual HER2-targeted therapy on the pCR and survival outcomes. Therefore, we conducted this meta-analysis of eligible RCTs to investigate the efficacy and safety of lapatinib plus trastuzumab, when compared with lapatinib or trastuzumab alone, for HER2-positive patients with breast cancer.

\section{MATERIALS AND METHODS}

\section{Search strategy}

This meta-analysis was conducted in accordance with the Preferred Reporting Items for Systematic Reviews and meta-analysis (PRISMA) criteria. ${ }^{17}$ PubMed, Embase and Web of Science databases from inception to 11 March 2016 were searched to identify relevant studies. The search was limited to human participants and no language restriction was imposed. Search terms included: ('breast neoplasms' (MeSH Terms) OR ('breast'(All Fields) AND 'neoplasms' (All Fields)) OR 'breast neoplasms' (All Fields) OR ('breast' (All Fields) AND 'cancer' (All Fields)) OR 'breast cancer' (All Fields)) AND ('lapatinib' (Supplementary Concept) OR 'lapatinib' (All Fields)) AND ('trastuzumab' (MeSH Terms) OR 'trastuzumab' (All Fields)). Details of the search strategy are shown in online supplementary appendix 1 . In addition, we also searched the reference lists of the included studies to identify other potentially eligible studies that we may leave out with our primary search.

\section{Study selection}

The following inclusive selection criteria were applied: (1) study design: RCT; (2) study population: female patients over the age of 18 who had histologically confirmed breast cancer with HER2-positive tumour; (3) comparison intervention: combination treatment of lapatinib with trastuzumab versus lapatinib or trastuzumab alone; (4) outcome measure: pCR, EFS, OS and adverse events. In case the same clinical trial appeared in several publications, we only included the most informative article or the longest follow-up study to avoid duplication of information.

\section{Data extraction and quality assessment}

We used a standardised data extraction sheet to extract data. The following information was obtained from each study: first author, publication year, number of patients in each arm, age of patients, population characteristics, the HRs with the corresponding 95\% CIs on EFS and OS and the risk ratios (RRs) with the corresponding 95\% CIs on the rate of pCR and incidence of adverse events. Data extraction was independently performed by two investigators (Z-qX and YZ), and discrepancies were resolved by discussion and consensus.

Two investigators (NL and P-jL) independently assessed the risk of bias in included RCTs with the method recommended by the Cochrane Collaboration. ${ }^{18}$ The quality of evidence for the outcome measures was evaluated using the Grading of Recommendations Assessment, Development and Evaluation (GRADE) approach. ${ }^{19}$

\section{Statistical analysis}

We estimated the pooled HR with $95 \%$ CI for time-to-event outcomes and the pooled RR with 95\% CI for dichotomous outcomes. A random-effects model ${ }^{20}$ was used to pool the estimates for all the outcomes. A fixed-effects model $^{21}$ was used when the heterogeneity among the included studies was zero. Heterogeneity across the studies was tested using the Cochran $\mathrm{Q}$ statistic and quantified with the $\mathrm{I}^{2}$ statistic, in which $\mathrm{I}^{2}>50 \%$ indicated significant heterogeneity. ${ }^{22}$ We also investigated the influence of a single study on the overall pooled estimate by deleting one study in each turn. Owing to the limited number $(<10)$ of included studies in each analysis, the assessment of publication bias was not performed. Pooled estimates with calculated $\mathrm{p}$ values smaller than 0.05 were declared statistically significant, except when otherwise stated. All statistical analyses were performed using STATA, V.12.0 (Stata Corporation, College Station, Texas, USA).

\section{RESULTS}

\section{Identification of eligible studies}

The initial search yielded 816 relevant citations from PubMed, Embase and Web of Science. Of these, 358 were excluded as duplicate records, and 440 were excluded after review of the title/abstract (figure 1). Therefore, 18 potential studies were identified for the final analysis; however, 11 studies were excluded because $2^{2324}$ were single-arm phase II studies, $6^{12} 25-29$ were unrelated to our topics, $1^{30}$ did not provide outcomes of our interest, $1^{31}$ was a non-RCT design and 1 was a 
Figure 1 Eligibility of studies for inclusion in meta-analysis.

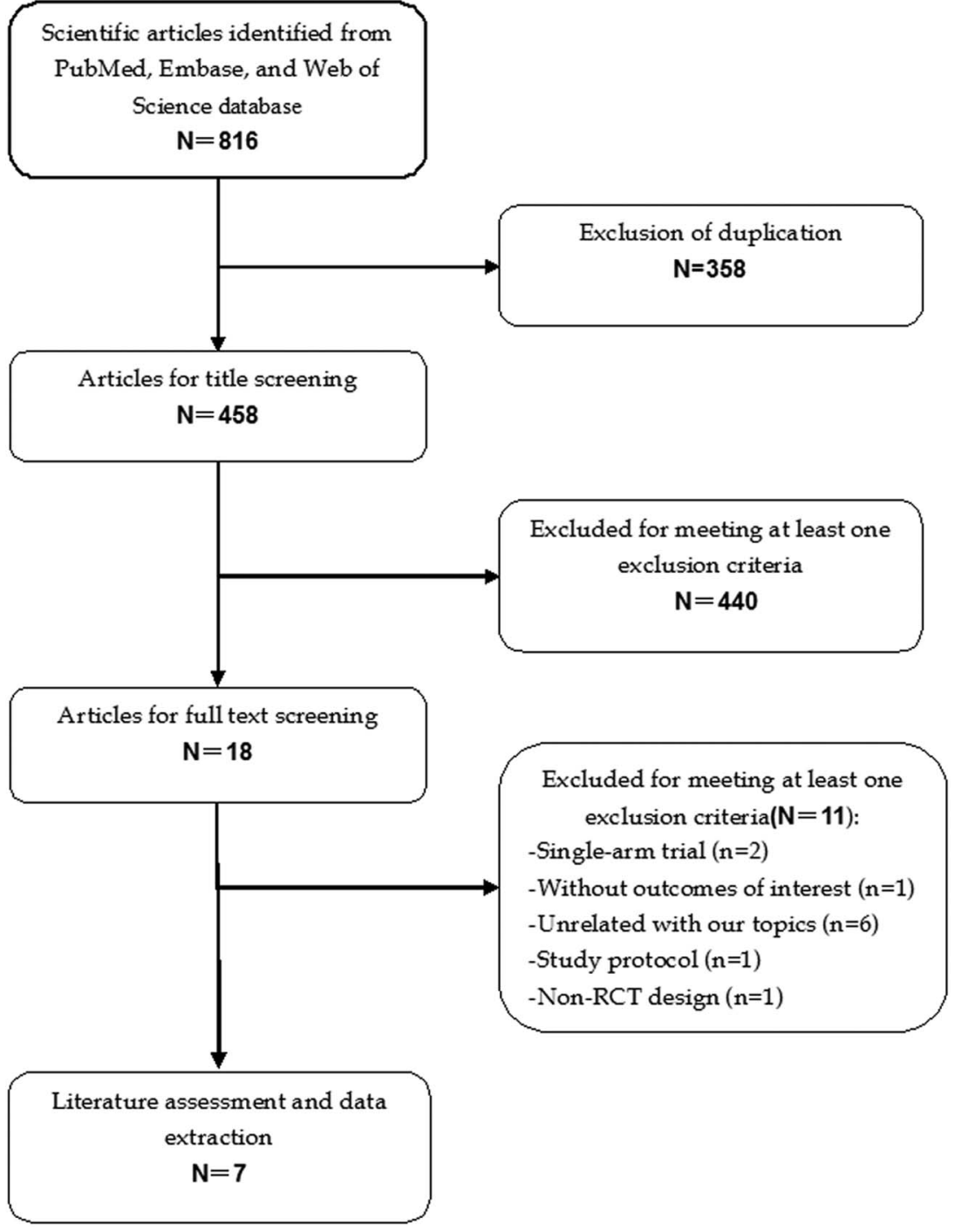

protocol study. ${ }^{32}$ Finally, seven RCTs (involving 2084 patients) ${ }^{15} \quad 16 \quad 33-37$ that met the inclusion criteria were included in this meta-analysis.

\section{Characteristics of eligible studies}

The main patient characteristics of the four included studies were presented in table 1. All seven included studies were well-performed, prospective RCTs. Clinical characteristics were matched for hormone receptor and clinical lymph-node status in each study. These studies were published between 2012 and 2015. Most of the patients in these studies were from Europe, North and South America, Asia and Africa. All these eligible patients were older than 18 years and had histologically or cytologically confirmed HER2-positive breast cancer. Among the seven studies, only one study enrolled patients who had prior trastuzumab-based therapies, ${ }^{34}$ whereas patients in the remaining studies had no previously treated experience.

The ALTTO study was initially reported by de Azambuja et al in 2014, ${ }^{33}$ and updated in 2016 by Sonnenblick et $a l^{38}$ However, the updated study only provided data in the subpopulations which developed early rash or not, but not in the overall population. Thus, we included the first version of the study published in 2014 and excluded the updated version.

The details of the risk-of-bias assessment are summarised in figure 2. Three trials were judged to be at low risk of bias, and four at unclear risk of bias. Three trials generated an adequate randomisation sequence and appropriate allocation concealment. The GRADE level of evidence was high for pCR, EFS and OS.

\section{Pathological complete response}

Five studies reported pCR in study patients. ${ }^{15} \quad 16 \quad 35-37$ Breast pCR was noted in 502 of $880(57.0 \%)$ patients in the combination group, and 349 of 855 (40.8\%) patients in the other therapy group. The pooled results using a random-effects model demonstrated that the pCR rate was significantly higher in the combination group than in the lapatinib or trastuzumab group $(\mathrm{RR}=1.43,95 \% \mathrm{CI}$ 1.23 to $1.67 ; \mathrm{p}<0.001$ ) (figure 3 ). There was moderate heterogeneity among the included individual studies ( $p$ for heterogeneity $=0.031 ; \mathrm{I}^{2}=51.0 \%$ ). 
Table 1 Baseline characteristics of patients in the trials included in the meta-analysis

\begin{tabular}{|c|c|c|c|c|c|}
\hline Study & Country & $\begin{array}{l}\text { Treatment } \\
\text { regimen }\end{array}$ & $\begin{array}{l}\text { Number of } \\
\text { patients }\end{array}$ & $\begin{array}{l}\text { Age } \\
\text { (range, years) }\end{array}$ & $\begin{array}{l}\text { Duration } \\
\text { (years) }\end{array}$ \\
\hline \multirow[t]{3}{*}{ Baselga et $a l^{15}$} & $\begin{array}{l}\text { Europe, Asia, North and South America, } \\
\text { and South Africa }\end{array}$ & $\begin{array}{l}\text { Lapatinib } \\
\text { +trastuzumab }\end{array}$ & 152 & $50(43-59)$ & 1.5 \\
\hline & & Lapatinib & 154 & $50(42-56)$ & \\
\hline & & Trastuzumab & 149 & $49(44-57)$ & \\
\hline \multirow[t]{3}{*}{ Robidoux et al ${ }^{16}$} & USA, Canada and Puerto Rico & $\begin{array}{l}\text { Lapatinib } \\
\text { +trastuzumab }\end{array}$ & 174 & $>18$ & 4.3 \\
\hline & & Lapatinib & 174 & $>18$ & \\
\hline & & Trastuzumab & 181 & $>18$ & \\
\hline \multirow[t]{3}{*}{$\begin{array}{l}\text { de Azambuja } \\
\text { et } a{ }^{\beta 3}\end{array}$} & $\begin{array}{l}\text { Europe, Asia, North and South America, } \\
\text { and South Africa }\end{array}$ & $\begin{array}{l}\text { Lapatinib } \\
\text { +trastuzumab }\end{array}$ & 152 & $50(43-59)$ & 3.77 \\
\hline & & Lapatinib & 154 & $50(42-56)$ & \\
\hline & & Trastuzumab & 149 & 49 (44-57) & \\
\hline \multirow[t]{2}{*}{ Blackwell et $a \beta^{\beta 4}$} & North America and Europe & $\begin{array}{l}\text { Lapatinib } \\
\text { +trastuzumab }\end{array}$ & 148 & $52(26-81)$ & 3 \\
\hline & & Lapatinib & 148 & $51(29-78)$ & 2.5 \\
\hline \multirow[t]{2}{*}{ Guarneri et $a{ }^{\beta 5}$} & Italy & $\begin{array}{l}\text { Lapatinib } \\
\text { +trastuzumab }\end{array}$ & 46 & $49(26-65)$ & 5 \\
\hline & & Lapatinib & 39 & 49 (34-68) & \\
\hline \multirow[t]{4}{*}{ Bonnefoi et $a l^{\beta 6}$} & UK & Trastuzumab & 36 & $50(34-65)$ & NR \\
\hline & & $\begin{array}{l}\text { Lapatinib } \\
\text { +trastuzumab }\end{array}$ & 52 & $49.4(27.3-70.8)$ & \\
\hline & & Lapatinib & 23 & $49.9(27.3-68.5)$ & \\
\hline & & Trastuzumab & 53 & 47 (25.3-68.9) & \\
\hline \multirow[t]{3}{*}{ Holmes et $a \beta^{\beta 7}$} & USA & $\begin{array}{l}\text { Lapatinib } \\
\text { +trastuzumab }\end{array}$ & 33 & $50.0(28-66)$ & NR \\
\hline & & Lapatinib & 34 & 52 (25-67) & \\
\hline & & Trastuzumab & 33 & $54(21-67)$ & \\
\hline
\end{tabular}

Subgroup analysis based on the treatment comparators was conducted. The pooled estimates using a random-effects model showed that a combination treatment of lapatinib and trastuzumab was associated with a significantly higher $\mathrm{pCR}$ rate than either lapatinib $(\mathrm{RR}=1.54,95 \%$ CI 1.16 to $2.03 ; \mathrm{p}=0.003)$ or trastuzumab alone $(\mathrm{RR}=1.36,95 \%$ CI 1.12 to $1.65 ; \mathrm{p}=0.002)$ (figure 3 ).

Subgroup analysis based on the hormone receptor status indicated that combination treatment significantly increased the pCR in patients with hormone receptorpositive $(\mathrm{RR}=1.31,95 \%$ CI 1.02 to $1.69 ; \mathrm{p}=0.034)$ or negative $(\mathrm{RR}=1.39,95 \%$ CI 1.14 to $1.69 ; \mathrm{p}=0.001)$ (figure 4). Moreover, for patients with tumour size $\leq 5$ / $>5 \mathrm{~cm}$, combination treatment significantly improved the pCR in patients irrespective of their tumour size (for tumour size $\leq 5 \mathrm{~cm}, \mathrm{RR}=1.65,95 \%$ CI $1.08,2.52 ; \mathrm{p}=0.020$; for tumour size $>5 \mathrm{~cm}, \mathrm{RR}=1.46,95 \%$ CI 1.05 to 2.04; $\mathrm{p}=0.025$ ) (figure 4).

\section{Event-free survival and overall survival}

Two RCTs reported EFS and OS in study patients. ${ }^{33} 34$ The pooled results of these studies using a fixed-effects model showed that combination treatment of lapatinib and trastuzumab significantly prolonged the EFS and OS more than did lapatinib or trastuzumab alone in patients with HER2-positive breast cancer (for EFS,
$\mathrm{HR}=0.75$, 95\% CI 0.60 to $0.93 ; \mathrm{p}=0.009$; for $\mathrm{OS}$, $\mathrm{HR}=0.72,95 \%$ CI 0.56 to $0.93 ; \mathrm{p}=0.011$ ) (figure 5 ). The test for heterogeneity was not significant (for EFS, $\mathrm{p}=0.0853 ; \mathrm{I}^{2}=0.0 \%$; for OS, $\mathrm{p}=0.649 ; \mathrm{I}^{2}=0.0 \%$ ).

\section{Adverse events}

Six studies included in this meta-analysis presented data on adverse events. ${ }^{15} 16$ 33-36 The most common adverse events were diarrhoea, rash or erythema, neutropenia and hepatic adverse events. Pooled analysis using a random-effects model showed that compared with monotherapy, combination therapy was associated with a significantly higher incidence of grade 3 or 4 diarrhoea $(\mathrm{RR}=6.38,95 \%$ CI 4.05 to $10.07 ; \mathrm{p}=0.001)$, rash or erythema $(\mathrm{RR}=1.51,95 \% \mathrm{CI} 1.06$ to $2.16 ; \mathrm{p}=0.0024)$, and hepatic adverse events $(\mathrm{RR}=2.31,95 \%$ CI 1.35 to 3.98; $\mathrm{p}=0.002)$.

\section{DISCUSSION}

The present study was a systematic review and meta-analysis of RCTs with the objective of comparing the combination treatment of lapatinib and trastuzumab to lapatinib or trastuzumab alone in the treatment of HER2-positive breast cancer. This study indicated that lapatinib in combination with trastuzumab significantly 


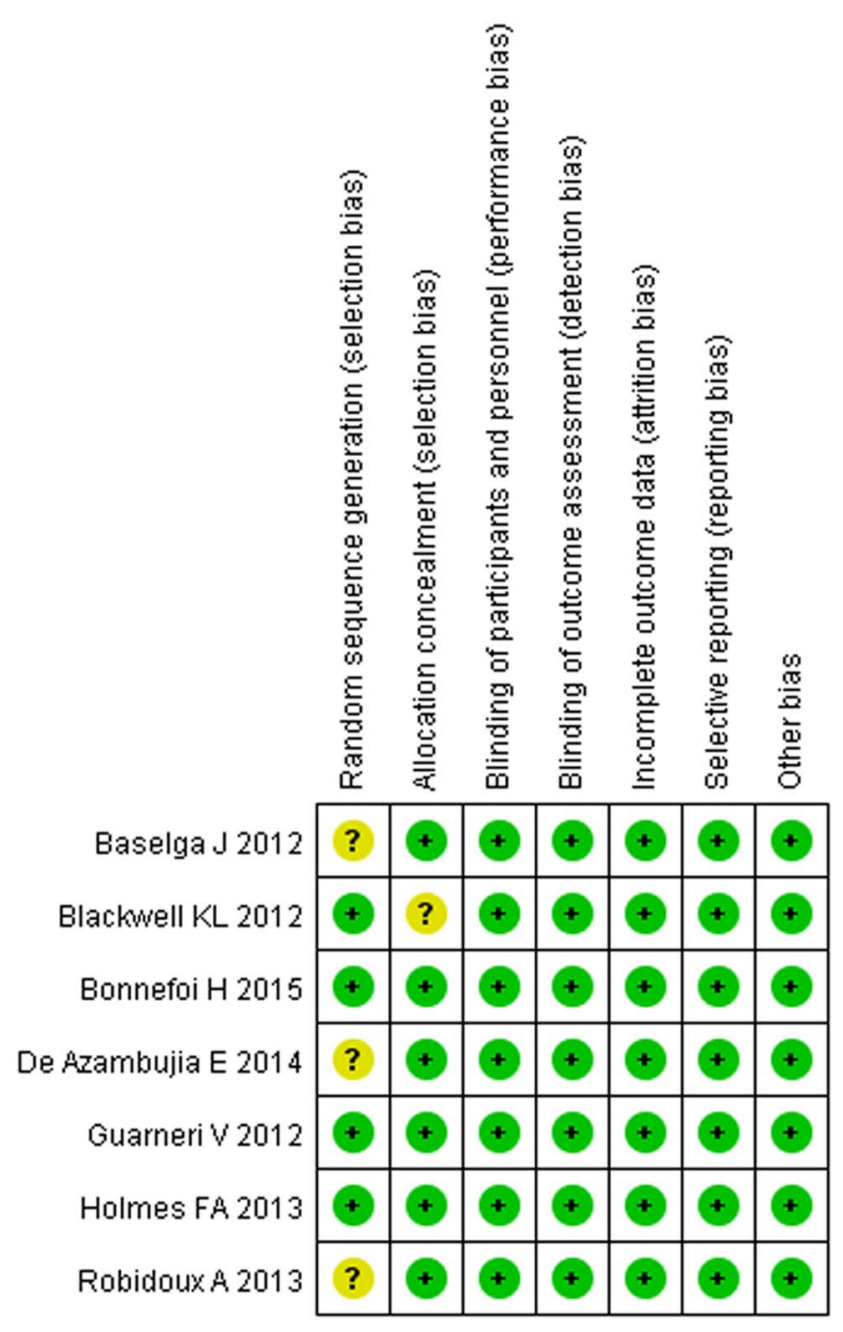

Figure 2 Risk of bias summary. increased pCR, and prolonged EFS and OS in the treatment of HER2-positive breast cancer. Moreover, the combination treatment increased pCR when it was compared with either lapatinib or trastuzumab alone. A significantly higher incidence of grade 3 or 4 adverse events, including diarrhoea, rash or erythema, and hepatic adverse events, were observed in the lapatinib plus trastuzumab group. Our study demonstrated that patients with HER2-positive breast cancer could achieve increased pCR, and improved EFS and OS when they were treated with the combination treatment of lapatinib and trastuzumab.

There has been one published meta-analysis of neoadjuvant dual HER2-targeted therapy with lapatinib and trastuzumab in the treatment of patients with early-stage HER2-positive breast cancer. ${ }^{39}$ In that study, the authors included four peer-review reports and one abstract to estimate the effect of adding lapatinib to trastuzumab plus neoadjuvant chemotherapy on pCR rate. ${ }^{39}$ Their results showed that the combination treatment was associated with a significant improvement in the pCR. ${ }^{39}$ Our meta-analysis expands on this early meta-analysis to provide better characteristics for the combination treatment for HER2-positive breast cancer. First, in our study, there are more enlarged sample sizes than in the previous study, which increases the statistical power to evaluate the effect of combination treatment. Second, we also assessed the effects of combination treatment in the subpopulation of patients with different hormone receptor status and tumour size, whereas in the previous study, the authors only evaluated the effect of combination treatment on the pCR. Furthermore, we also were capable of evaluating the effects of combined therapy in
Figure 3 Pathological complete response for combination therapy of lapatinib and trastuzumab with lapatinib or trastuzumab alone.

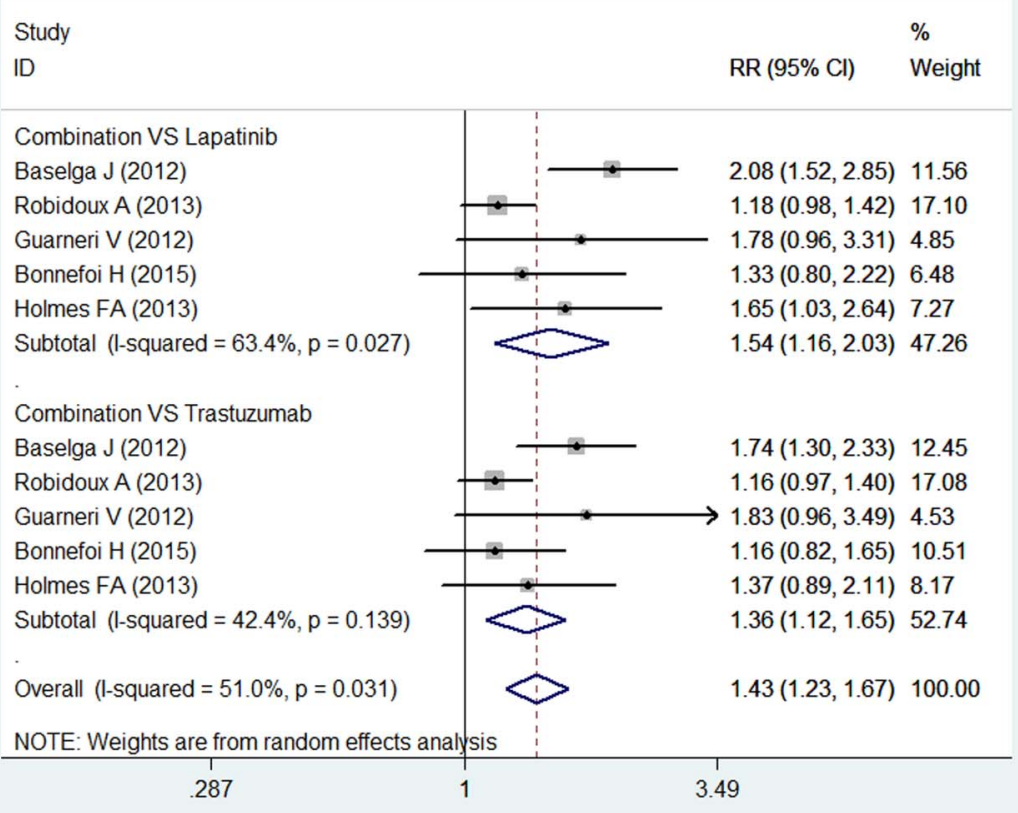




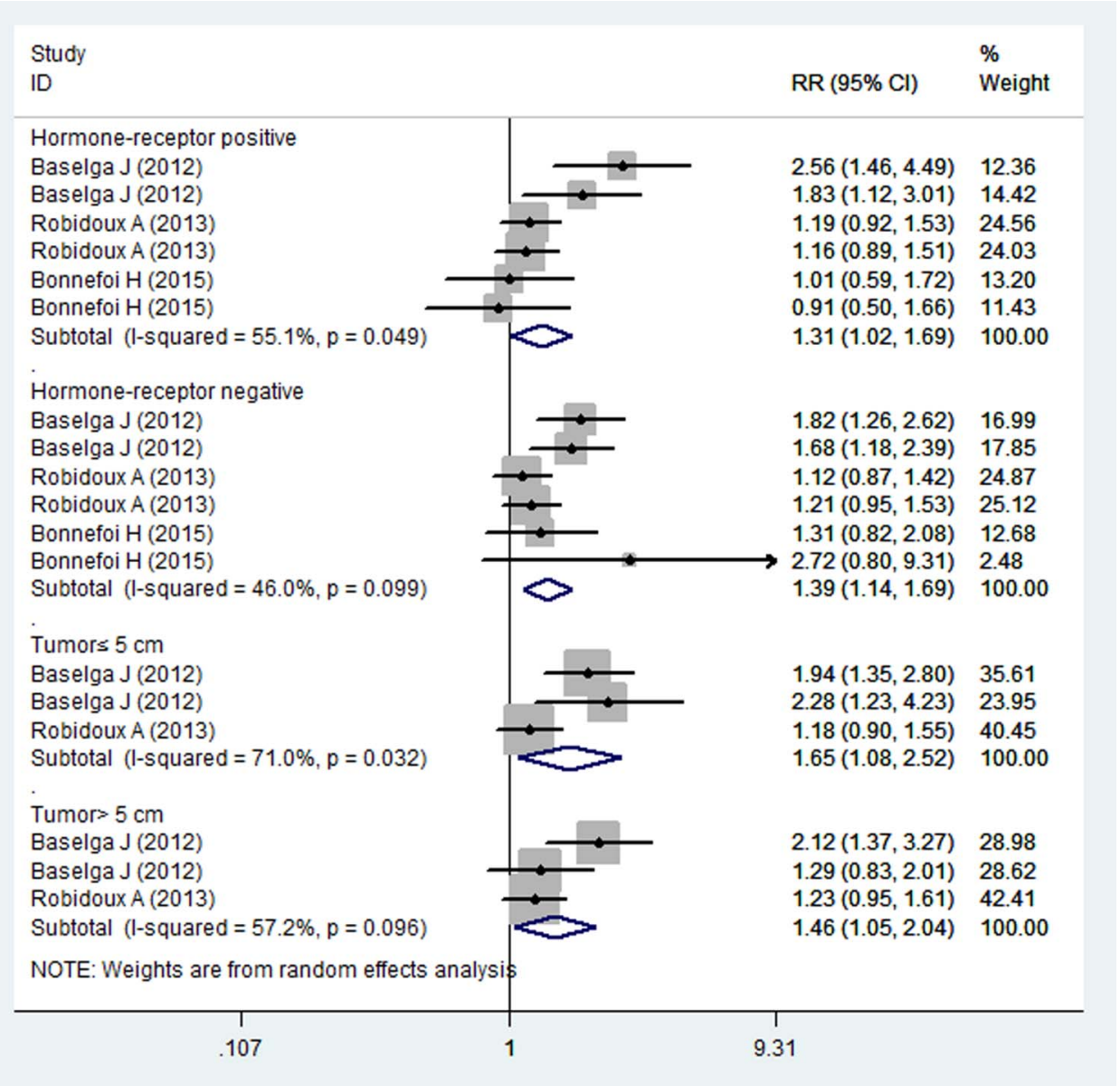

Figure 4 Pathological complete response for combination therapy of lapatinib and trastuzumab with lapatinib or trastuzumab alone in the subgroup populations.

the survival outcomes (EFS and OS), which has not been investigated in the previous meta-analysis.

On the basis of the previous meta-analysis, we furthermore included other studies. ${ }^{33} 34{ }^{36}$ With the added statistical power, this study demonstrated that the combination of lapatinib and trastuzumab significantly increased the pCR more than did lapatinib or trastuzumab alone, which was in line with previous meta-analysis. Moreover, in the subgroup analysis, the combination treatment also increased the pCR irrespective of hormone receptor status and tumour size.

In this meta-analysis, the clinical response rate of lapatinib plus trastuzumab was superior to lapatinib or trastuzumab alone. Also, we noted that this higher pCR rate for the combination treatment was clear across all subgroups tested. This result was consistent with other studies of combined therapy in HER2-positive tumours. The NeoALTTO study, ${ }^{15}$ in which patients with HER2-positive early breast cancer were randomly allocated to receive lapatinib or trastuzumab, or lapatinib in combination with trastuzumab, showing a doubling in pCR in the combination group than in the lapatinib group. The pCR rate in the hormone-receptor-positive patients was $16.25 \%$ in the lapatinib group compared with $41.56 \%$ in the combination group. ${ }^{15}$ For the patients with a hormone-receptor-negative tumour, the pCR rate in these two groups was $33.78 \%$ and $61.33 \%$, respectively. ${ }^{15}$ Investigators of the NSABP protocol B-41 study also reported a higher pCR rate in the combination treatment. ${ }^{16}$ In that study, the pCR in hormone-receptor-positive and negative tumours was highest in the combination group $(55.6 \%$ and $73.0 \%$, respectively), intermediate in the trastuzumab group (46.7\% and $65.5 \%$, respectively) and lowest in the lapatinib group $\left(48.0 \%\right.$ and $60.6 \%$, respectively). ${ }^{16}$ Despite the combination of lapatinib and trastuzumab significantly increased pCR in hormone-receptor-positive and negative patients, the rate of pCR seemed to be slightly higher in hormone-receptor-negative patients.

With regard to the survival outcomes of EFS and OS, our data demonstrated that combination treatment significantly prolonged the EFS and OS. Our findings are similar to the recently reported EGF 104900 trial, ${ }^{34}$ in which the combination treatment demonstrated a significant 4.5-month median OS and 3-week EFS advantage in patients with heavily pretreated HER2-positive metastatic breast cancer. ${ }^{34}$ These results confirmed the preclinical data that lapatinib in combination with trastuzumab has 


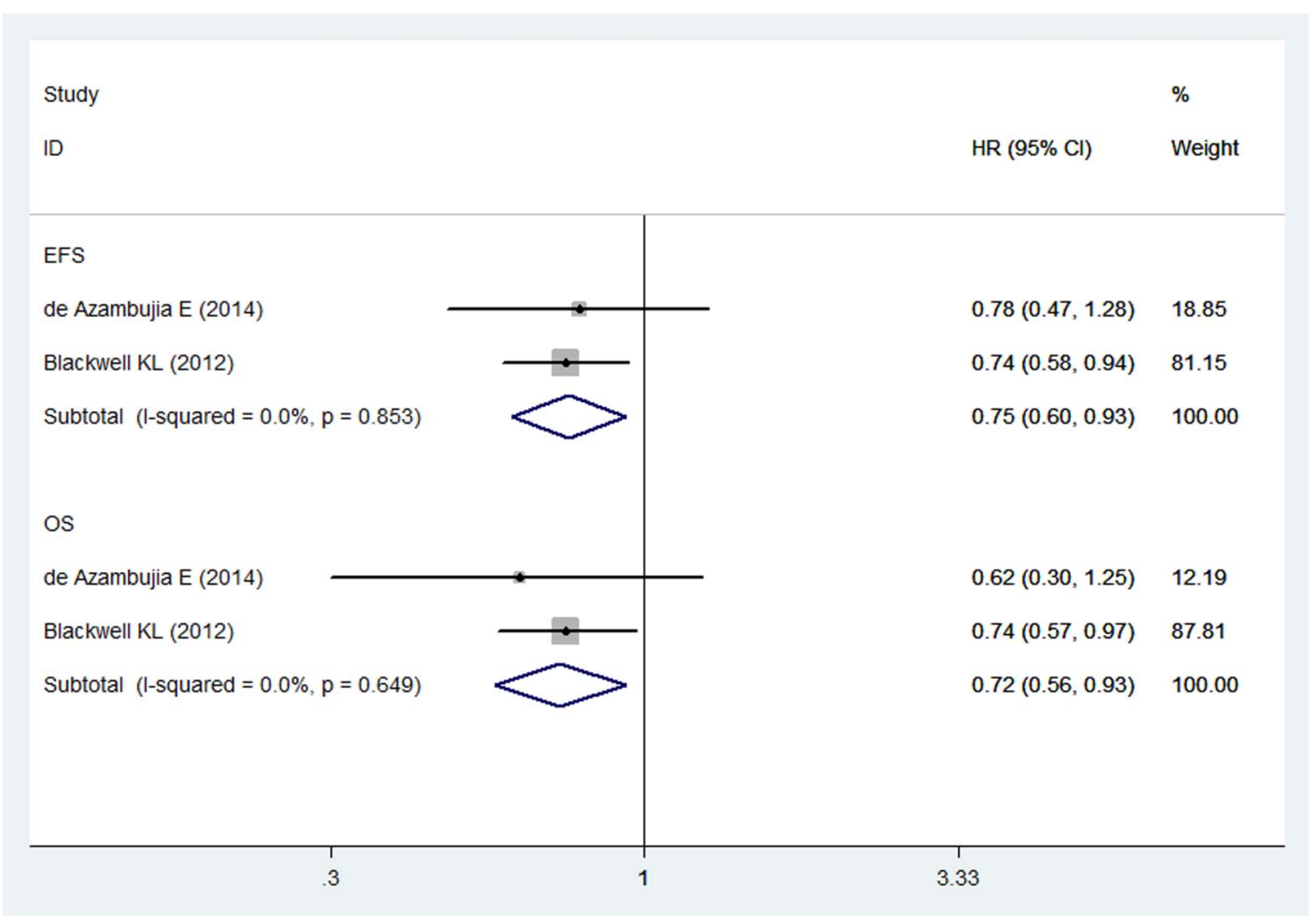

Figure 5 Event-free survival and overall survival for combination therapy of lapatinib and trastuzumab with lapatinib or trastuzumab alone.

synergistic antiproliferative effects. ${ }^{14}$ In the NeoALTTO trial, ${ }^{33}$ however, the survival advantage of the combination treatment was not observed. In that study, patients with HER2-positive early breast cancer were randomly allocated to the lapatinib group (154), trastuzumab group (149) or lapatinib plus trastuzumab group (152). ${ }^{33}$ At the end of follow-up, the 3-year EFS and 3-year OS were $84 \%$ and $95 \%$ in the combination group, $78 \%$ and $93 \%$ in the lapatinib group and $76 \%$ and $90 \%$ in the trastuzumab group, respectively. ${ }^{33}$ The differences between these groups were not significant. However, for patients who achieve pCR after the combination treatment, they had significantly better EFS and OS than did those without pCR.

The ALTTO trial is an international, intergroup, openlabel, phase III randomised trial in patients with HER2-positive early breast cancer. ${ }^{40}$ In that study, 2093 and 2097 patients were randomly assigned to the combination and trastuzumab groups, respectively. After a median follow-up of 4.5 years, patients in the combination group had a $16 \%$ reduction in DFS compared with those in the trastuzumab group ( $\mathrm{HR}=0.84,95 \%$ CI 0.70 to 1.02$).{ }^{40}$ However, the difference did not reach statistical significance $(p=0.048)$. This result is inconsistent with the finding of the EGF104900 trial, $^{34}$ which observed a beneficial effect of combination treatment. In the EGF104900 trial, ${ }^{34} 146$ and 145 patients were randomly assigned to the combination and lapatinib arms. The median PFS for patients in the two groups was 11.1 and 8.1 weeks, respectively. ${ }^{34}$ Patients in the combination group experienced a significantly improved PFS compared with those in the lapatinib group ( $\mathrm{HR}=0.74,95 \%$ CI 0.58 to $0.94 ; \mathrm{p}=0.011$ ).

The conflicting results between the ALTTO and EGF104900 trials could be explained by the following possible reasons: first, the patients' characteristics might account for the results. Patients enrolled in the EGF104900 trial were from America (62.5\%) and Europe $(37.5 \%),{ }^{34}$ whereas patients in the ALTTO trial were Caucasian $(69 \%)$ and Asian $(26 \%) .{ }^{40}$ Patients from different races may have a different response to the combination treatment. Second, the difference in drug dosage and administration schedule between the two trials might explain the results. In the EGF104900 trial, ${ }^{34}$ patients received intravenous trastuzumab $2 \mathrm{mg} / \mathrm{kg}$ weekly in combination with $1500 \mathrm{mg}$ daily oral lapatinb, or oral lapatinb $1500 \mathrm{mg}$ daily monotherapy, whereas in the ALTTO trial, ${ }^{40}$ the trastuzumab was administered with a loading dose of $4 \mathrm{mg} / \mathrm{kg}$ once and then $2 \mathrm{mg} / \mathrm{kg}$ weekly during chemotherapy, or at a loading dose of $8 \mathrm{mg} / \mathrm{kg}$ once and then $6 \mathrm{mg} / \mathrm{kg}$ every 3 weeks when given alone. Lapatinb was administered with a dosage of $750 \mathrm{mg}$ /day in the combination treatment. Third, a difference in the sample size might influence results. In the EGF104900 trial, ${ }^{34}$ the sample size for the combination arm and trastuzumab arm was 146 and 145, respectively, whereas in the ALTTO trial, ${ }^{40}$ the corresponding figures were 2093 and 2097, respectively. Studies with a small sample size 
are more likely to result in an overestimation of the treatment effect compared with large sample studies. Thus, the beneficial effect of combination therapy in the EGF104900 trial might be overestimated.

The association between pCR and the survival outcomes was also reported in several previous studies. ${ }^{7} 4142$ In the NOAH study, patients with HER2-positive locally advanced or inflammatory breast cancer were randomly assigned to the trastuzumab and chemotherapy group or chemotherapy group. ${ }^{7}$ Results from that study showed that there was a strong correlation between pCR and the improved EFS. ${ }^{7}$ The investigators of the TECHNO study $^{42}$ also reported an association between pCR and prolonged disease-free survival (DFS) and OS. In that study, the 3-year DFS was $88 \%$ in patients with pCR compared with $73 \%$ in patients without $\mathrm{pCR}(\mathrm{p}=0.01)$; the 3-year OS was $96 \%$ in patients with pCR compared with $86 \%$ in patients without pCR $(\mathrm{p}=0.025){ }^{42}$ Another meta-analysis of 11955 patients with primary breast cancer $^{43}$ also demonstrated that patients who achieved pCR had improved EFS and OS, and the association between pCR and long-term survival outcomes was strongest in patients with triple-negative breast cancer and in those with HER2-positive, hormone-receptor-negative tumours treated with trastuzumab. ${ }^{43}$

Regarding the safety profile, our study showed that the incidence of grade 3 or 4 toxicities, including diarrhoea, rash or erythema, neutropenia and hepatic adverse events, was higher in the combination group than in the monotherapy group. In a previously published trial, early rash was reported to be associated with a higher chance of pCR, mainly in patients older than 50 years (odds risk, OR=3.76, 95\% CI 1.69 to 8.34 ), but not in those $<50$ years $(\mathrm{OR}=0.92,95 \%$ CI 0.45 to 1.88 ; $\mathrm{p}$ for interaction $=0.01) .{ }^{44}$ However, no significant correlation was observed between pCR and diarrhoea or hepatic adverse events. ${ }^{44}$

There are some potential limitations in this meta-analysis. First, our meta-analysis is based on seven RCTs and some of them have a relatively modest sample size, which may lead to an overestimation of the treatment effect when compared with studies with larger samples. Second, the patient characteristics, including hormone receptor status, tumour size, clinical status of lymph nodes and treatment regimens, vary greatly across studies. All of these factors may cause the heterogeneity of our analysis and have a potential impact on our final results. Third, our exploration of the survival effects of lapatinib plus trastuzumab in patients with HER2-positive breast cancer was insufficient because of the sparse reporting among the included studies. Finally, it should be noted that all of these trials were partly funded by the pharmaceutical industry, and their results might have been affected by the inherent conflict of interest and possible bias.

In summary, our meta-analysis indicated that a combination of lapatinib and trastuzumab can significantly improve pCR, EFS and OS in the treatment of
HER2-positive breast cancer. Moreover, it also induced an acceptable and tolerable risk of diarrhoea, rash, neutropenia and hepatic adverse events. However, considering the potential bias and confounding of the included studies, further larger scale, well-designed RCTs are needed to identify these findings.

Contributors $Z-q X$ and $Y Z$ conceived the study aims and design and contributed to the systematic review and data extraction. NL, P-jL, LG, XG and $\mathrm{X}$-jT performed the analysis, interpreted the results and drafted the manuscript. All authors contributed to the revision of the data extraction, interpretation of results and to the review of the manuscript.

Funding This research received no specific grant from any funding agency in the public, commercial or not-for-profit sectors.

Competing interests None declared.

Provenance and peer review Not commissioned; externally peer reviewed.

Data sharing statement No additional data are available.

Open Access This is an Open Access article distributed in accordance with the Creative Commons Attribution Non Commercial (CC BY-NC 4.0) license, which permits others to distribute, remix, adapt, build upon this work noncommercially, and license their derivative works on different terms, provided the original work is properly cited and the use is non-commercial. See: http:// creativecommons.org/licenses/by-nc/4.0/

\section{REFERENCES}

1. Slamon DJ, Godolphin W, Jones LA, et al. Studies of the HER-2/neu proto-oncogene in human breast and ovarian cancer. Science 1989;244:707-12

2. Owens MA, Horten BC, Da Silva MM. HER2 amplification ratios by fluorescence in situ hybridization and correlation with immunohistochemistry in a cohort of 6556 breast cancer tissues. Clin Breast Cancer 2004;5:63-9.

3. Slamon DJ, Leyland-Jones B, Shak S, et al. Use of chemotherapy plus a monoclonal antibody against HER2 for metastatic breast cancer that overexpresses HER2. N Engl J Med 2001;344:783-92.

4. Perez EA, Romond EH, Suman VJ, et al. Four-year follow-up of trastuzumab plus adjuvant chemotherapy for operable human epidermal growth factor receptor 2-positive breast cancer: joint analysis of data from NCCTG N9831 and NSABP B-31. J Clin Oncol 2011;29:3366-73.

5. Goldhirsch A, Gelber RD, Piccart-Gebhart MJ, et al. 2 years versus 1 year of adjuvant trastuzumab for HER2-positive breast cancer (HERA): an open-label, randomised controlled trial. Lancet 2013;382:1021-8.

6. Buzdar AU, Ibrahim NK, Francis D, et al. Significantly higher pathologic complete remission rate after neoadjuvant therapy with trastuzumab, paclitaxel, and epirubicin chemotherapy: results of a randomized trial in human epidermal growth factor receptor 2-positive operable breast cancer. J Clin Oncol 2005;23:3676-85.

7. Gianni L, Eiermann W, Semiglazov V, et al. Neoadjuvant chemotherapy with trastuzumab followed by adjuvant trastuzumab versus neoadjuvant chemotherapy alone, in patients with HER2-positive locally advanced breast cancer (the NOAH trial): a randomised controlled superiority trial with a parallel HER2negative cohort. Lancet 2010;375:377-84.

8. Geyer CE, Forster J, Lindquist D, et al. Lapatinib plus capecitabine for HER2-positive advanced breast cancer. $N$ Engl J Med 2006;355:2733-43.

9. Burstein HJ, Storniolo AM, Franco S, et al. A phase II study of lapatinib monotherapy in chemotherapy-refractory HER2-positive and HER2-negative advanced or metastatic breast cancer. Ann Oncol 2008;19:1068-74.

10. Blackwell KL, Pegram MD, Tan-Chiu E, et al. Single-agent lapatinib for HER2-overexpressing advanced or metastatic breast cancer that progressed on first- or second-line trastuzumab-containing regimens. Ann Oncol 2009;20:1026-31.

11. Blackwell KL, Burstein HJ, Storniolo AM, et al. Randomized study of Lapatinib alone or in combination with trastuzumab in women with ErbB2-positive, trastuzumab-refractory metastatic breast cancer. J Clin Oncol 2010;28:1124-30. 
12. Cameron D, Casey M, Press M, et al. A phase III randomized comparison of lapatinib plus capecitabine versus capecitabine alone in women with advanced breast cancer that has progressed on trastuzumab: updated efficacy and biomarker analyses. Breast Cancer Res Treat 2008;112:533-43.

13. Konecny GE, Pegram MD, Venkatesan N, et al. Activity of the dual kinase inhibitor lapatinib (GW572016) against HER-2-overexpressing and trastuzumab-treated breast cancer cells. Cancer Res 2006;66:1630-9.

14. Scaltriti M, Verma C, Guzman M, et al. Lapatinib, a HER2 tyrosine kinase inhibitor, induces stabilization and accumulation of HER2 and potentiates trastuzumab-dependent cell cytotoxicity. Oncogene 2009;28:803-14.

15. Baselga J, Bradbury I, Eidtmann $\mathrm{H}$, et al. Lapatinib with trastuzumab for HER2-positive early breast cancer (NeoALTTO): a randomised, open-label, multicentre, phase 3 trial. Lancet 2012;379:633-40.

16. Robidoux A, Tang G, Rastogi $P$, et al. Lapatinib as a component of neoadjuvant therapy for HER2-positive operable breast cancer (NSABP protocol B-41): an open-label, randomised phase 3 trial. Lancet Oncol 2013;14:1183-92.

17. Moher D, Liberati A, Tetzlaff J, et al. Preferred reporting items for systematic reviews and meta-analyses: the PRISMA statement. Ann Intern Med 2009;151:264-9, w64.

18. Higgins J, Green S. Cochrane handbook for systematic reviews of interventions version 5.1.0 [updated march 2011]. The cochrane collaboration. 2011. http://www.Cochrane-handbook.Org.

19. Guyatt GH, Oxman AD, Vist GE, et al. GRADE: an emerging consensus on rating quality of evidence and strength of recommendations. BMJ 2008;336:924-6.

20. Mantel N, Haenszel W. Statistical aspects of the analysis of data from retrospective studies of disease. J Natl Cancer Inst 1959;22:719-48.

21. DerSimonian R, Laird N. Meta-analysis in clinical trials. Control Clin Trials 1986;7:177-88.

22. Higgins JP, Thompson SG. Quantifying heterogeneity in a meta-analysis. Stat Med 2002;21:1539-58.

23. Storniolo AM, Pegram MD, Overmoyer B, et al. Phase I dose escalation and pharmacokinetic study of lapatinib in combination with trastuzumab in patients with advanced ErbB2-positive breast cancer. J Clin Oncol 2008;26:3317-23.

24. Rimawi MF, Mayer IA, Forero A, et al. Multicenter phase II study of neoadjuvant lapatinib and trastuzumab with hormonal therapy and without chemotherapy in patients with human epidermal growth factor receptor 2-overexpressing breast cancer: TBCRC 006. $J$ Clin Oncol 2013;31:1726-31.

25. Iwata $\mathrm{H}$, Fujii $\mathrm{H}$, Masuda N, et al. Efficacy, safety, pharmacokinetics and biomarker findings in patients with HER2-positive advanced or metastatic breast cancer treated with lapatinib in combination with capecitabine: results from 51 Japanese patients treated in a clinical study. Breast Cancer 2015;22:192-200.

26. Alba E, Albanell J, de la Haba J, et al. Trastuzumab or lapatinib with standard chemotherapy for HER2-positive breast cancer: results from the GEICAM/2006-14 trial. Br J Cancer 2014;110:1139-47.

27. Welslau M, Dieras V, Sohn JH, et al. Patient-reported outcomes from EMILIA, a randomized phase 3 study of trastuzumab emtansine (T-DM1) versus capecitabine and lapatinib in human epidermal growth factor receptor 2-positive locally advanced or metastatic breast cancer. Cancer 2014;120:642-51.

28. Gelmon KA, Boyle FM, Kaufman B, et al. Lapatinib or trastuzumab plus taxane therapy for human epidermal growth factor receptor 2-positive advanced breast cancer: final results of NCIC CTG MA.31. J Clin Oncol 2015;33:1574-83.

29. Pivot X, Manikhas A, Zurawski B, et al. CEREBEL (EGF111438): a phase III, randomized, open-label study of lapatinib plus capecitabine versus trastuzumab plus capecitabine in patients with human epidermal growth factor receptor 2-positive metastatic breast cancer. J Clin Oncol 2015;33:1564-73.

30. Wu Y, Amonkar MM, Sherrill BH, et al. Impact of lapatinib plus trastuzumab versus single-agent lapatinib on quality of life of patients with trastuzumab-refractory HER2+ metastatic breast cancer. Ann Oncol 2011;22:2582-90.

31. Esteva FJ, Franco SX, Hagan MK, et al. An open-label safety study of lapatinib plus trastuzumab plus paclitaxel in first-line HER2-positive metastatic breast cancer. Oncologist 2013;18:661-6.

32. Guarneri V, Frassoldati A, Piacentini F, et al. Preoperative chemotherapy plus lapatinib or trastuzumab or both in HER2-positive operable breast cancer (CHERLOB Trial). Clin Breast Cancer 2008;8:192-4.

33. de Azambuja E, Holmes AP, Piccart-Gebhart M, et al. Lapatinib with trastuzumab for HER2-positive early breast cancer (NeoALTTO): survival outcomes of a randomised, open-label, multicentre, phase 3 trial and their association with pathological complete response. Lancet Oncol 2014;15:1137-46.

34. Blackwell KL, Burstein HJ, Storniolo AM, et al. Overall survival benefit with lapatinib in combination with trastuzumab for patients with human epidermal growth factor receptor 2-positive metastatic breast cancer: final results from the EGF104900 Study. J Clin Oncol 2012;30:2585-92.

35. Guarneri V, Frassoldati A, Bottini A, et al. Preoperative chemotherapy plus trastuzumab, lapatinib, or both in human epidermal growth factor receptor 2-positive operable breast cancer: results of the randomized phase II CHER-LOB study. J Clin Oncol 2012;30:1989-95.

36. Bonnefoi $\mathrm{H}$, Jacot $\mathrm{W}$, Saghatchian $\mathrm{M}$, et al. Neoadjuvant treatment with docetaxel plus lapatinib, trastuzumab, or both followed by an anthracycline-based chemotherapy in HER2-positive breast cancer: results of the randomised phase II EORTC 10054 study. Ann Oncol 2015;26:325-32.

37. Holmes FA, Espina V, Liotta LA, et al. Pathologic complete response after preoperative anti-HER2 therapy correlates with alterations in PTEN, FOXO, phosphorylated Stat5, and autophagy protein signaling. BMC Res Notes 2013;6:507.

38. Sonnenblick A, de Azambuja E, Agbor-Tarh D, et al. Lapatinib-related rash and breast cancer outcome in the ALTTO phase III randomized trial. J Natl Cancer Inst 2016;108:pii: djw037

39. Hicks M, Macrae ER, Abdel-Rasoul M, et al. Neoadjuvant dual HER2-targeted therapy with lapatinib and trastuzumab improves pathologic complete response in patients with early stage HER2-positive breast cancer: a meta-analysis of randomized prospective clinical trials. Oncologist 2015;20:337-43.

40. Piccart-Gebhart M, Holmes E, Baselga J, et al. Adjuvant lapatinib and trastuzumab for early human epidermal growth factor receptor 2-positive breast cancer: results from the randomized phase III adjuvant lapatinib and/or trastuzumab treatment optimization trial. $J$ Clin Oncol 2016;34:1034-42.

41. Untch M, Fasching $\mathrm{P}$, Konecny $\mathrm{G}$. Pathological complete response after neoadjuvant chemotherapy+trastuzumab treatment predicts survival and detects a patient subgroup at high need for improvement of anti-HER2 therapy: three year median follow-up data of the TECHNO Trial. Cancer Res 2011;70(Suppl 2):P1-11-03.

42. Untch M, Fasching PA, Konecny GE, et al. Pathologic complete response after neoadjuvant chemotherapy plus trastuzumab predicts favorable survival in human epidermal growth factor receptor 2-overexpressing breast cancer: results from the TECHNO trial of the AGO and GBG study groups. J Clin Oncol 2011;29:3351-7.

43. Cortazar P, Zhang L, Untch M, et al. Pathological complete response and long-term clinical benefit in breast cancer: the CTNeoBC pooled analysis. Lancet 2014;384:164-72.

44. Azim HA Jr, Agbor-Tarh D, Bradbury I, et al. Pattern of rash, diarrhea, and hepatic toxicities secondary to lapatinib and their association with age and response to neoadjuvant therapy: analysis from the NeoALTTO trial. J Clin Oncol 2013;31:4504-11. 